\title{
POTENCIAL ENERGÉTICO DA MADEIRA DE Eucalyptus sp. EM FUNÇÃO DA IDADE E DE DIFERENTES MATERIAIS GENÉTICOS ${ }^{1}$
}

\author{
Angélica de Cássia Oliveira Carneiro² ${ }^{2}$, Ana Flávia Neves Mendes Castro ${ }^{3}$, Renato Vinícius Oliveira \\ Castro $^{4}$, Rosimeire Cavalcante dos Santos ${ }^{5}$, Lumma Papaspyrou Ferreira ${ }^{6}$, Renato Augusto Pereira \\ Damásio $^{3}$ e Benedito Rocha Vital ${ }^{2}$
}

\begin{abstract}
RESUMO - Este trabalho teve como objetivo determinar a influência da idade e de diferentes materiais genéticos de Eucalyptus sp. na produção de madeira para energia. Neste estudo, foram avaliados três clones de Eucalyptus sp., em quatro idades diferentes, aos 3, 4, 5 e 7 anos, sendo provenientes da Gerdau S/A. De cada árvore foram retirados cinco discos $(0 \%, 25 \%, 50 \%, 75 \%$ e $100 \%$ da altura comercial do tronco), bem como determinados a densidade básica, o poder calorífico superior e a análise elementar da madeira, e, como base nesses valores, foi possível estimar a quantidade de energia m-3. Observou-se que houve efeito da idade e do material genético para a densidade básica da madeira e para o poder calorífico superior. Verificou-se ainda que a quantidade de energia m-3 aumentou com a idade, e houve diferença significativa entre os três materiais genéticos avaliados. O clone GG 680 apresentou melhor desempenho dessa variável, apresentando, aos 7 anos, 2.943 kW.h.m-33. Cabe ressaltar que a escolha do melhor material genético deve levar em consideração as características tecnológicas da madeira, bem como a sua produtividade e efetividade técnica de produção.
\end{abstract}

Palavras-chave: Eucalyptus sp.; Potencial energético; Clones; Idade.

\section{POTENTIAL ENERGY OF Eucalyptus sp. WOOD ACCORDING TO AGE AND DIFFERENT GENETIC MATERIALS}

\begin{abstract}
The present study aimed to determine the influence of age and different genetic material of Eucalyptus sp. in wood energy production. Therefore, we evaluated three Eucalyptus sp. clones from Gerdau S/A, at four different ages: 3, 4, 5 and 7 years. Five discs were removed from each tree $(0 \%, 25 \%, 50 \%$, $75 \%, 100 \%$ of the trunk commercial height), and the wood basic density and calorific value were determined. Based on these values we estimated the energy m-3 amount. We observed an effect of age and genetic material for the wood density and the calorific value. Moreover, the amount of energy m-3 increased with increasing age, and the three genetic materials evaluated were significantly different between them. The GG 680 clone showed better performance for this variable, presenting at seven years 2,943 kW.h.m. $m^{-3}$. Note that the selection of the best genetic material must consider the wood technological characteristics, as well as their productivity and effectiveness of technical production.
\end{abstract}

Keywords: Eucalyptus sp.; Energy potential; Clones; Age.

\footnotetext{
${ }^{1}$ Recebido em 21.03.2013 aceito para publicação em 11.03.2014.

${ }^{2}$ Departamento de Engenharia Florestal, Universidade Federal de Viçosa, UFV, Brasil. E-mail: <cassiacarneiro@ufv.br>e <bvital@ufv.br>.

${ }^{3}$ Programa de Pós-Graduação em Ciência Florestal, Universidade Federal de Viçosa, UFV, Brasil. E-mail: <mendesafn@hotmail.com> e <renatodamasio@gmail.com>.

${ }^{4}$ Departamento de Ciências Agrárias, Universidade Federal de São João Del Rey, UFSJ, Campus Sete Lagoas, Brasil. E-mail: <castrorvo@ymail.com>.

${ }^{5}$ Unidade Acadêmica de Jundiai, Universidade Federal do Rio Grande do Norte, UFRN, Brasil. E-mail: <meire_caico@yahoo.com.br> .

${ }^{6}$ Comunicação Corporativa \& Marketing, Sociedade de Investigações Florestais, SIF. E-mail: <lummapf@gmail.com>.
} 


\section{INTRODUÇÃO}

A madeira é largamente utilizada pelo homem para diversas finalidades, entre elas o uso para fins energéticos. Com o aumento da utilização de combustíveis fósseis, o uso da madeira para produção de energia diminuiu. Entretanto, atualmente, devido à pressão de órgãos ambientais e de organizações não governamentais para que ocorra diminuição na emissão de poluentes, existe maior busca por combustíveis provenientes de fontes renováveis, que poluam menos, e a utilização da biomassa tem ganhado forças nesse cenário.

Do ponto de vista energético, biomassa é todo recurso renovável oriundo de material orgânico, seja vegetal ou animal, que pode ser utilizado para produção de energia (ANEEL, 2011).

A biomassa, no Brasil, é constituída, em grande parte, pela madeira e, quando se avalia a produção de energia, pode-se dizer que seu uso se divide em produção de carvão vegetal (carbonização) e consumo direto da lenha (combustão) (VALE et al., 2002).

Para queima direta, é melhor utilizar madeiras com maior poder calorífico, pois essa propriedade está relacionada com o rendimento energético que, por sua vez, está relacionado com a sua constituição química, em que os teores de celulose, hemiceluloses, lignina, extrativos e substâncias minerais variam de uma espécie para outra (QUIRINO et al., 2005). Além disso, pode-se dizer que a densidade da madeira também é uma característica bastante relevante, pois é um dos índices de qualidade da madeira mais importantes, uma vez que está correlacionada diretamente com a produção de massa seca, com as propriedades físico-mecânicas e pode ser facilmente determinada (PALERMO et al., 2004), além de se relacionar com a qualidade dos produtos (SANTOS, 2010).

A idade das árvores também interfere consideravelmente, pois diversas transformações ocorrem na madeira à medida que envelhece, podendo-se citar variações nas suas composições químicas, físicas e anatômicas. Aidade de uma floresta é fator muito importante para uma empresa, uma vez que há mudança nas propriedades da madeira com o aumento da sua idade.

De maneira geral, as empresas estipulam uma idade de corte com base na produtividade volumétrica do povoamento, desconsiderando a variação da qualidade da madeira que ocorre em função da idade da floresta.
Portanto, é necessário conhecer as propriedades da madeira e a sua variação conforme a idade, para destiná-la a determinado uso, a fim de promover seu melhor aproveitamento. Dessa forma, este trabalho teve como objetivo determinar a influência da idade e de diferentes materiais genéticos de Eucalyptus sp. na produção de madeira para energia.

\section{MATERIAL E MÉTODOS}

\subsection{Dados}

Neste trabalho, foram utilizados três clones de Eucalyptus sp. (Tabela 1), provenientes de plantios comerciais, pertencentes à Gerdau S.A., localizado no Município de Lassance, MG. Foram selecionadas três árvores de diâmetro médio para cada um dos 12 tratamentos, totalizando 36 árvores (amostras). As árvores foram coletadas em plantios comerciais com espaçamento médio de 3,5 m × 2,5 m, nas classes de idade de 3, 4, 5 e 7 anos.

\subsection{Preparo do material}

De cada árvore, foram retirados cinco discos, correspondentes a $0 \%, 25 \%, 50 \%, 75 \%$ e $100 \%$ da altura comercial do tronco, sendo a densidade básica da madeira obtida para cada disco, ou seja, sentido base-topo, enquanto para o poder calorífico e para a análise química elementar foram utilizadas amostras compostas.

\subsection{Determinação da densidade básica da madeira}

De cada disco foram retiradas duas cunhas opostas, passando pela medula, as quais foram identificadas e destinadas à determinação da densidade básica.

Tabela 1 - Identificação do material genético utilizado (Família: Myrtaceae; Gênero: Eucalyptus).

Table 1 - Identification of the genetic material used (Family: Myrtaceae; Gender: Eucalyptus).

\begin{tabular}{lcc}
\hline Clones & Idades (anos) & Material genético \\
\hline GG 100 & 3 & E. urophylla $\times$ E. grandis \\
& 4 & E. urophylla $\times$ E. grandis \\
& 5 & E. urophylla $\times$ E. grandis \\
& 7 & E. urophylla $\times$ E. grandis \\
\hline GG 157 & 3 & E. urophylla \\
& 4 & E. urophylla \\
& 5 & E. urophylla \\
\hline GG 680 & 3 & E. urophylla \\
& 4 & E. urophylla $\times$ E. grandis \\
& 5 & E. urophylla $\times$ E. grandis \\
& 7 & E. urophylla $\times$ E. grandis \\
\hline
\end{tabular}


Os procedimentos utilizados para a análise estão de acordo com o método de imersão em água, descrito por Vital (1984). Os valores foram calculados a partir da média aritmética das densidades das respectivas cunhas.

\subsection{Determinação do poder calorífico superior}

O poder calorífico superior da madeira foi determinado de acordo com a metodologia descrita pela norma daABNTNBR 8633(ABNT, 1984), utilizando-se uma bomba calorimétrica adiabática. As amostras de madeira foram transformadas em serragem, utilizando-se um moinho de laboratório tipo Willey, de acordo com a norma TAPPI 257 om-52 (TAPPI, 1998). Foi utilizada a fração que passou pela peneira $n^{\circ} 16$ internacional, com malha de 40 mesh, e ficou retida na peneira $\mathrm{n}^{\circ} 24$ internacional, com malha de 60 mesh (ASTM, 1982). As amostras foram secas em estufa a $103 \pm 2^{\circ} \mathrm{C}$ até massa constante, para determinação do poder calorífico superior.

\subsection{Determinação da composição elementar}

Na análise elementar (carbono, nitrogênio, hidrogênio, enxofre e oxigênio), utilizou-se uma massa equivalente a 2,5 mg $( \pm 0,5)$ de serragem seca, selecionada em peneiras sobrepostas de 200 e 270 mesh, utilizando-se a fração retida na peneira de 270 mesh. O equipamento utilizado foi o Vario Micro Cube CHNS-O. Esse aparelho pode atingir temperaturas de até $1.200^{\circ} \mathrm{C}$ e proporciona a combustão da serragem. Ocorrem, então, reações químicas que geram gases, que, por sua vez, são conduzidos aos tubos redutores, presentes em um compartimento específico. Em seguida, os elementos químicos são individualizados numa sequência induzida, de acordo com a massa molecular de cada um, e quantificados a partir de um software específico. O valor de oxigênio foi quantificado pelo somatório do C, N, H e S decrescido de 100.

\subsection{Estimativa de energia}

A massa seca de madeira de cada clone foi obtida a partir da densidade básica $\left(\mathrm{kg} \mathrm{m}^{-3}\right)$ encontrada em $1 \mathrm{~m}^{3}$ de madeira. Para o cálculo da energia por $\mathrm{m}^{3}$ de madeira, expressa em kcal, multiplicou-se a massa seca da madeira (kg) pelo respectivo poder calorífico superior de cada clone, conforme apresentado a seguir:

$$
\text { Energia }\left(\mathrm{kcal} \mathrm{m}^{-3}\right)=\mathrm{DB} \times \mathrm{PCS}
$$

em que energia $\left(\mathrm{kcal} \mathrm{m}^{-3}\right)$ = produção energética por metro cúbico de madeira; $\mathrm{DB}$ = massa seca da madeira em kg; e PCS = poder calorífico superior da madeira em kcal kg-1.

Para conversão da energia em $\mathrm{kcal} / \mathrm{m}^{3}$ para kW.h.m ${ }^{-3}$, dividiram-se os valores em $\mathrm{kcal} \mathrm{m}^{-3}$ por 860 , conforme Santos (2010).

\section{RESULTADOS}

Na Figura 1 estão apresentados os valores médios da densidade básica da madeira em função dos tratamentos idade e clone. De maneira geral, as madeira de Eucalyptus sp., usadas para produção de carvão vegetal, apresentam densidade básica, classificada como densidade média, em torno de $0,54 \mathrm{~g} \mathrm{~cm}^{-3}$ (BRITO et al., 1983).

Neste trabalho, observou-se aumento significativo da densidade básica da madeira com o incremento da idade (Figura 1), sendo somente aos 7 anos observados valores semelhantes aos valores ideais para produção de carvão vegetal, conforme estipulado por Brito et al. (1983) $\left(0,52 \mathrm{~g} \mathrm{~cm}^{-3} ; 0,54 \mathrm{~g} \mathrm{~cm}^{-3} ; 0,56 \mathrm{~g} \mathrm{~cm}^{-3}\right.$, para GG 100, GG 157 e GG 680, respectivamente).

Na Figura 2 estão apresentados os valores médios do poder calorífico superior da madeira em função dos tratamentos, da idade e do clone.

O poder calorífico superior da madeira não apresentou homogeneidade de variância, portanto foi realizada uma análise não paramétrica, aplicando-se o teste de Kruskall-Wallis.

Médias seguidas da mesma letra maiúscula entre as idades e minúscula entre clones não diferem entre si, a 5\% de significância, pelo teste de Tukey.

Means followed by the same upper case letter between the ages and lower case among clones do not differ at 5\% significance level by the test of Tukey.

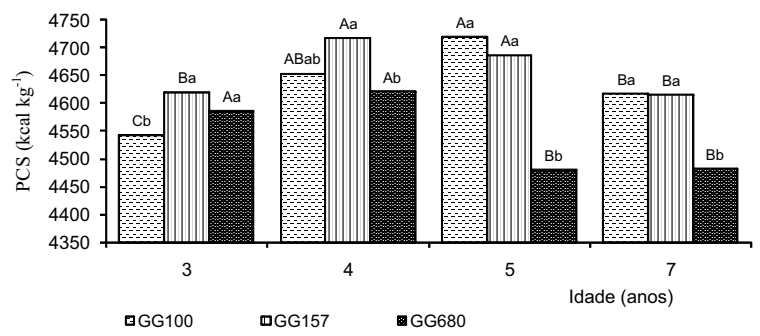

Figura 1 - Densidade básica da madeira em função da idade e do clone.

Figure 1 - Wood basic density as a function of age and clone.

Revista Árvore, Viçosa-MG, v.38, n.2, p.375-381, 2014 
Médias seguidas da mesma letra maiúscula entre as idades e minúscula entre os clones não diferem entre si, todos a 5\% de significância, pelo teste de Kruskall-Wallis.

Means followed by the same upper case letter between the ages and lower case among clones do not differ at 5\% significance level by the test of Kruskall-Wallis.

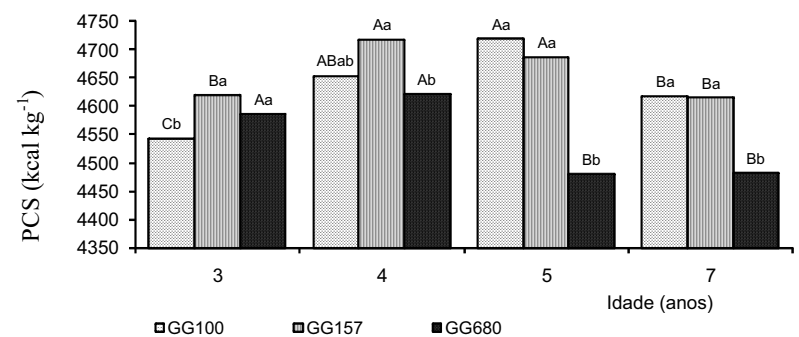

Figura 2 - Poder calorífico superior $\left(\mathrm{kcal} \mathrm{kg}^{-1}\right)$ da madeira em função da idade e do clone.

Figure 2 - Higher Calorific Value $\left(\mathrm{kcal} \mathrm{kg}^{-1}\right)$ of wood as a function of age and clone.

Analisando a Figura 2, observa-se que houve efeito da idade no poder calorífico superior da madeira, e diferença significativa entre os clones, em todas as idades. Os valores médios de poder calorífico superior da madeira, obtidos neste trabalho, foram de $4.633 \mathrm{kcal} \mathrm{kg}^{-1}$ (GG 100), $4.660 \mathrm{kcal} \mathrm{kg}^{-1}$ (GG 157) e $4.542 \mathrm{kcal} \mathrm{kg}^{-1}$ (GG 680).

Quando se utiliza biomassa para energia, a composição elementar é uma propriedade do combustível muito importante e constitui a base para análise do processo de combustão. De maneira geral, a composição elementar da madeira não varia muito, e os valores médios são: 50\% de carbono; 6\% de hidrogênio; 43\% de oxigênio; 0,15\% de nitrogênio; e 1\% de cinzas (RAAD, 2004).

Na Figura 3 estão apresentados os valores médios para análise elementar da madeira dos três clones em estudo, nas diferentes idades avaliadas.

Analisando a Figura 3a, observa-se que não houve influência da idade no teor de nitrogênio, exceto para o clone GG 680, que apresentou menores teores desse elemento nas idades de 5 e 7 anos. Entre os clones ocorreu diferença significativa em todas as idades, exceto aos 4 anos.

Analisando a Figura 3b, observa-se que não houve influência da idade no teor de carbono, exceto no clone GG 680, que apresentou aumento no teor de carbono com a idade. Também, verifica-se que houve efeito significativo do clone nas diferentes idades avaliadas, exceto na idade de 4 anos. Ainda, constata-se que não houve influência da idade no teor de hidrogênio, exceto no clone GG 100. Entre os clones, não houve diferença significativa, exceto aos 3 anos.

Ainda na Figura 3, letra d, verifica-se que não houve influência da idade no teor de oxigênio, independentemente do clone. Entre os clones não ocorreu diferença significativa nas idades de 3 e 4 anos, sendo a diferença aos 5 e 7 anos significativa.

O teor de enxofre da madeira não apresentou homogeneidade de variância, portanto foi realizada uma análise não paramétrica, aplicando-se o teste de Kruskall-Wallis.

De maneira geral, pela Figura 3e, verifica-se que houve efeito da idade no teor de enxofre dos clones GG 157 e GG 680. Houve também diferença significativa entre os clones, exceto na idade de 5 anos. No clone GG 680, nas idades de 5 e 7 anos, o teor de enxofre foi zero.

Na Figura 4 são apresentados os valores médios da quantidade de energia por $\mathrm{m}^{3}$ em função dos tratamentos, da idade e do clone.

\section{DISCUSSÃO}

Analisando a Figura 1, verifica-se que houve diferença significativa, na densidade básica da madeira, entre os clones em todas as idades, e o clone GG 680 apresentou valores médios superiores nas quatro idades analisadas. Os valores de densidade básica da madeira variaram de $0,45 \mathrm{~g} \mathrm{~cm}^{-3}$ a $0,52 \mathrm{~g} \mathrm{~cm}^{-3} ; 0,45 \mathrm{~g} \mathrm{~cm}^{-3}$ a 0,54 $\mathrm{g} \mathrm{cm}^{-3} ; 0,49 \mathrm{~g} \mathrm{~cm}^{-3}$ a 0,56 $\mathrm{g} \mathrm{cm}^{-3}$, para GG 100, GG 157 e GG 680, respectivamente. Esses valores foram semelhantes aos observados na literatura (OLIVEIRA et al., 2010; SANTOS, 2010). No trabalho desenvolvido por Trugilho et al. (1996), também se verificou tendência de aumento na densidade básica da madeira com a idade e na madeira de Eucalyptus saligna, nas idades de 1, 2, 3 e 4 anos.

A densidade básica da madeira está diretamente relacionada com a produção de energia, ou seja, quanto maior a densidade, maior a quantidade de energia estocada por metro cúbico. Portanto, essa característica é muito importante para a escolha de espécies para queima direta da madeira. Além disso, a densidade da madeira mais elevada resulta em maiores densidades e resistência do carvão, bem como maior quantidade de massa enfornada, reduzindo os custos de produção 
Médias seguidas da mesma letra maiúscula entre as idades e minúscula entre clones não diferem entre si, a 5\% de significância, pelo teste de Tukey; (e) médias seguidas da mesma letra maiúscula entre as idades e minúscula entre clones não diferem entre si, a 5\% de significância, pelo teste de Kruskall-Wallis.

Means followed by the same upper case letter between the ages and lower case among clones do not differ at 5\% significance level by the test of Tukey; (e) Means followed by the same upper case letter between the ages and lower case among clones do not differ at $5 \%$ significance level by the test of Kruskall-Wallis.

(a)

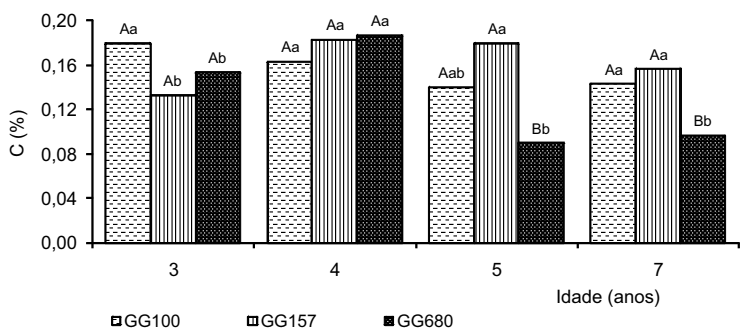

(c) (b)

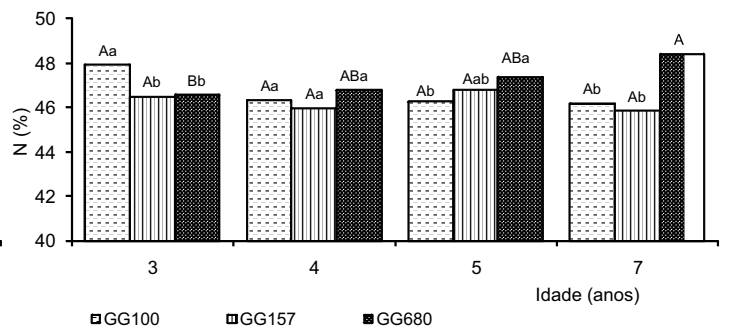

(d)

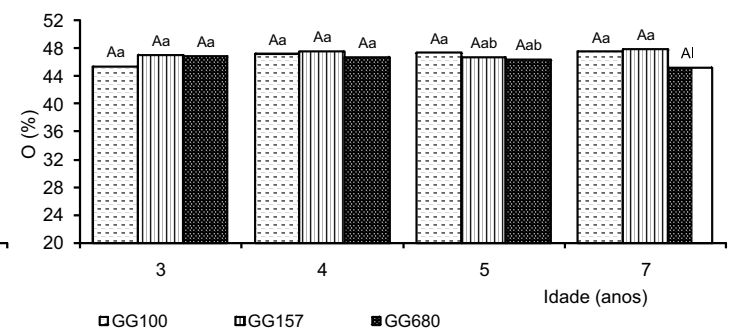

(e)

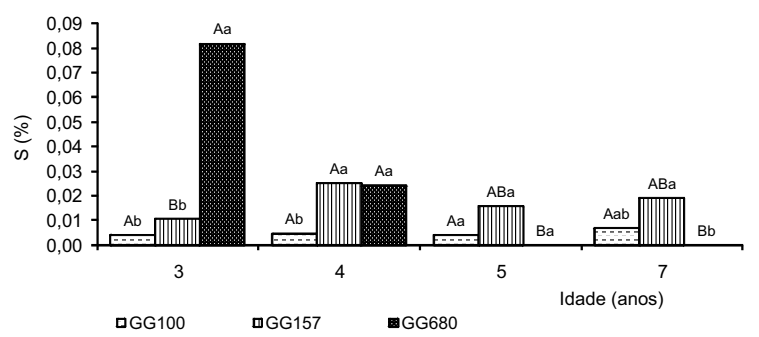

Figura 3 - Análise elementar da madeira em função da idade e do clone, sendo: (a) teor de nitrogênio (N\%); (b) teor de carbono (C\%); (c) teor de hidrogênio (H\%); (d) teor de oxigênio (O\%); e (e) teor de enxofre (S\%).

Figure 3 - Wood elementary analysis as a function of age and clone, being: (a) nitrogen content (N\%), (b) carbon content $(\% \mathrm{C}),(\mathrm{c})$ hydrogen content $(\mathrm{H} \%),(d)$ oxygen content $(\mathrm{O} \%)$, and (e) sulfur content $(\mathrm{S} \%)$.

e aumentando a produtividade das unidades de produção de carvão (UPC's) e nos altos-fornos (BRITO, 1993).

Valores semelhantes da variável de poder calorífico foram obtidos por Oliveira et al. (2010), que avaliaram um clone de Eucalyptus pellita na idade de 5 anos
(4.630 kcal kg-1); e Santos (2010), que estudou quatro clones híbridos de Eucalyptus sp. aos 7 anos (entre $4.274 \mathrm{kcal} \mathrm{kg}^{-1}$ e $4.585 \mathrm{kcal} \mathrm{kg}^{-1}$ ). Santana (2009) observou pouca influência da idade nessa variável e obteve valor médio de $4.610 \mathrm{kcal} \mathrm{kg}^{-1}$ de um clone de Eucalyptus grandis dos 3 aos 7 anos.

Revista Árvore, Viçosa-MG, v.38, n.2, p.375-381, 2014 
Médias seguidas da mesma letra maiúscula entre as idades e minúscula entre clones não diferem entre si, a 5\% de significância, pelo teste de Tukey.

Means followed by the same upper case letter between the ages and lower case among clones do not differ at 5\% significance level by the test of Tukey.

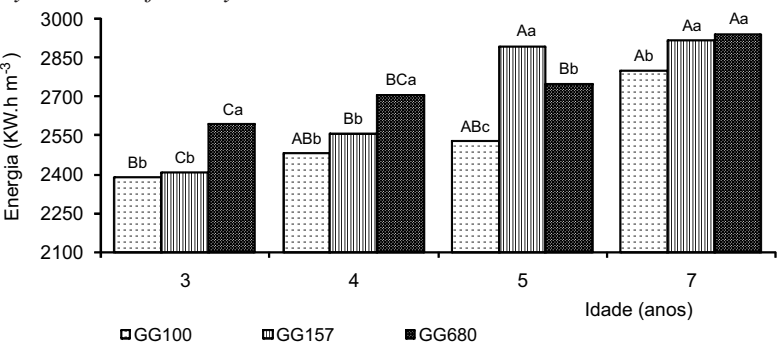

Figura 4-Quantidade de energia produzida por $\mathrm{m}^{3}$ em função da idade e do clone.

Figure 4-Amount of energy produced per $m^{3}$ as a function of age and clone.

O poder calorífico é uma das principais variáveis usadas para seleção de espécies com melhores características para fins energéticos, uma vez que está relacionada com a quantidade de energia liberada pela madeira durante a sua queima. A quantidade de calor desprendida da madeira é muito importante para se conhecer a capacidade energética de determinada espécie (SANTOS, 2010).

O uso da madeira como combustível requer a caracterização elementar, visando ao conhecimento das quantidades presentes de nitrogênio, pois altas concentrações desse elemento têm impacto negativo no meio ambiente e na saúde humana, devido à emissão do óxido nítrico produzido durante o processo de combustão (SANTANA, 2009).

O teor de carbono é muito importante, tanto para a produção de carvão vegetal quanto para a queima direta da madeira. Na queima direta, ele é totalmente consumido, enquanto na produção de carvão vegetal o carbono é convertido em carbono fixo, sendo esse o principal responsável pela energia estocada no carvão.

O hidrogênio libera, durante a queima, mais energia do que o carbono. Mesmo estando presente na madeira em pequenas quantidades, o teor de hidrogênio apresenta grande importância na geração de energia. Entretanto, durante a produção de carvão vegetal, à medida que se degrada a madeira, ocorrem a concentração de carbono e decréscimo nos teores de nitrogênio e hidrogênio; acima de $500^{\circ} \mathrm{C}$, acontece a perda excessiva de hidrogênio, devido à fase de dissociação desses elementos, não se observando aumento do poder calorífico superior, mesmo com a concentração de carbono.
Ao contrário do carbono e do hidrogênio, o oxigênio contribui negativamente para o poder calorífico. Assim, compostos com maiores teores de oxigênio implicam menor energia armazenada.

O enxofre, juntamente com as cinzas, é considerado as principais impurezas dos combustíveis (QUIRINO et al., 2005). Uma vez que a madeira apresenta baixos teores de enxofre, torna-se uma vantagem ambiental comparativa em relação aos combustíveis fósseis, como o carvão mineral (BRITO; BARRICHELO, 1979).

A combustão do enxofre gera o dióxido de enxofre $\left(\mathrm{SO}_{2}\right)$, que pode combinar-se com a água formando ácido sulfúrico diluído (QUIRINO et al., 2005). Assim como acontece com o nitrogênio, a presença de enxofre é prejudicial para o meio ambiente e para a saúde humana.

Analisando a Figura 4, observa-se que a quantidade de energia armazenada em $1 \mathrm{~m}^{3}$ de madeira aumentou com a idade, seguindo a mesma tendência da densidade básica da madeira. O clone GG 100 apresentou os menores valores dessa variável, observando-se uma variação de 2.389 kW.h.m- ${ }^{3}$ a 2.801 kW.h.m- ${ }^{3}$, enquanto o clone GG 680 apresentou o melhor desempenho dessa variável, e os valores médios variaram de 2.594 kW.h.m- ${ }^{3}$ a 2.943 kW.h.m- ${ }^{3}$. Os valores médios do clone GG 157 oscilaram entre 2.408 kW.h.m- ${ }^{3}$ e 2.916 kW.h.m- ${ }^{3}$.

Os valores observados neste estudo foram próximos aos resultados obtidos por Santos (2010), que obteve valores variando de 2.537 kW.h.m- ${ }^{3}$ a 2.926 kW.h.m- ${ }^{3}$ na quantidade de energia $\mathrm{m}^{-3}$ de clones de Eucalyptus sp. aos 7 anos. Entretanto, esses valores foram superiores aos encontrados por Rocha (2011), que, ao estudar um clone híbrido de Eucalyptus grandis $\times$ Eucalyptus camaldulensis, nas idades de 4, 5, 6 e 7 anos, obteve valores médios de energia $\mathrm{m}^{-3}$ variando de $1.821 \mathrm{~kW} . \mathrm{h}^{-\mathrm{m}^{-3}}$ a 2.464 kW.h.m ${ }^{-3}$.

\section{CONCLUSÕES}

Com base nos resultados, pode-se concluir-se que:

- A idade influencia a densidade básica da madeira e, consequentemente, a quantidade de energia armazenada por $\mathrm{m}^{3}$.

- O clone GG 680 apresentou melhor desempenho quando se avaliou o uso da madeira para geração de energia, atingindo, aos 7 anos, o valor de 2.802 kW.h.m-3. 
- O clone GG680 apresentou maiores teores de carbono em relação aos outros clones, em todas as idades, e, além disso, houve elevação desses teores com o aumento da idade.

- Maiores teores de carbono e de densidade básica, principalmente aos 7 anos, são importantes dados que contribuíram, significativamente, com os melhores resultados de poder calorífico, apresentados pelo clone GG680.

- Cabe ressaltar que a escolha do melhor material genético deve levar em consideração as características tecnológicas da madeira, bem como a sua produtividade e efetividade técnica de produção. Portanto, deve-se avaliar, também, a produtividade das florestas, com o objetivo de determinar se o ganho em qualidade de madeira é superior ao ganho pela produtividade.

\section{AGRADECIMENTOS}

À Fundação de Amparo à Pesquisa do Estado de Minas Gerais (FAPEMIG), SECTES e CNPq, pelo financiamento do Projeto; à Gerdau, pela doação da madeira; à Embrapa Floresta; e aos funcionários do Laboratório de Painéis e Energia da Madeira (LAPEM/UFV).

\section{REFERÊNCIAS}

AGÊNCIA NACIONAL DE ENERGIA ELÉTRICA ANNEL. Disponível em: <http://

www.aneel.gov.br/aplicacoes/atlas/pdf/05Biomassa(2).pdf>

ASSOCIAÇÃO BRASILEIRA DE NORMAS TÉCNICAS - ABNT. NBR 8633: carvão vegetal: determinação do poder calorífico. Rio de Janeiro: 1984. 13p.

AMERICAN SOCIETY FOR TESTING AND MATERIALS - ASTM. Standard methods of evaluating properties of wood-base fiber and particles

materials. Philladelphia: 1982.

BRITO, J. O. Reflexões sobre a qualidade do carvão vegetal para uso siderúrgico. Piracicaba: IPEF, 1993. (Circular Técnica, 181)

BRITO, J. O. et al. Análise da produção energética e de carvão vegetal de espécies de eucalipto. IPEF, n.23, p.53-56, 1983.

OLIVEIRA, A. C. et al. Parâmetros de qualidade da madeira e do carvão vegetal de Eucalyptus pellita F. Muell. Scientia Forestalis, v.38, n.87, p.431-439, 2010.
PALERMO, G. P. M. et al. Determinação da densidade da madeira de Pinus elliottii Englm, através de atenuação de radiação gama comparada a métodos tradicionais. Floresta e Ambiente, v.11, n.1, p.1-6, 2004.

QUIRINO, W. F. et al. Poder calorífico da madeira e de materiais ligno-celulósicos. Revista da Madeira, n.89, p.100-106, 2005.

RAAD, T. J. Simulação do processo de secagem e carbonização de Eucalyptus spp. 2004. 114f. Tese (Doutorado em Engenharia Mecânica) - Universidade Federal de Minas Gerais, Belo Horizonte, MG, 2004.

ROCHA, M. F. V. Influência do espaçamento e da idade na produtividade da madeira de Eucalyptus grandis $\mathrm{x}$ Eucalyptus camaldulensis para energia. 2011. 84f. Dissertação (Mestrado em Ciência Florestal) - Universidade Federal de Viçosa, Viçosa, MG, 2011.

SANTANA, W. M. S. Crescimento, produção e propriedades da madeira de um clone de Eucalyptus grandis e Eucalyptus urophylla com enfoque energético. 2009. 104f. Dissertação (Mestrado em Ciência e Tecnologia da Madeira) Universidade Federal de Lavras, Lavras, MG, 2009.

SANTOS, R. C. Parâmetros de qualidade da madeira e do carvão vegetal de clones de eucalipto. 2010. 173f. Tese (Doutorado em Ciência e Tecnologia da Madeira) Universidade Federal de Lavras, Lavras, MG, 2010.

TAPPI TECHNICAL DIVISIONS AND COMMITTEES. TAPPI test methods. Atlanta: 1998. 46 p.

TRUGILHO, P. F.; LIMA, J. T.; MENDES, L. M. Influência da idade nas características físicoquímicas e anatômicas da madeira de Eucalyptus saligna. Cerne, v.2, n.1, p.15, 1996.

VALE, A. T.; BRASIL, M. A. M.; LEÃO, A. L. Quantificação e caracterização energética da madeira e casca de espécies do cerrado. Ciência Florestal, v.12, n.1, p.71-80, 2002.

VITAL, B. R. Métodos de determinação de densidade da madeira. Viçosa, MG: SIF, 1984. 21p. (Boletim Técnico, 1).

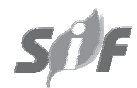

Revista Árvore, Viçosa-MG, v.38, n.2, p.375-381, 2014 
